\section{Ein Gegenbeispiel zur Theorie der schwachen Konvergenz}

\author{
von
}

J. SCHREIER (Lwów).

Herr Banach und Herr Saks haben folgenden Satz für den Raum der mit der $p$-ten Potenz $(p>1)$ integrablen Funktionen bewiesen:

Jede schwach konvergente Folge enthält eine Teilfolge, deren arithmetische Mittel stark konvergieren ${ }^{1}$ ).

Herr B an ach und Herr Saks haben nun die Frage gestellt, ob ein analoger Satz für den Raum der stetigen Funktionen gültig ist ${ }^{2}$ ).

Es ist mir gelungen diese Frage negativ zu beantworten.

In' Anbetracht der üblichen Definition der schwachen und starken Konvergenz im Raume der stetigen Funktionen genügt es dazu eine Funktionenfolge $\left\{f_{i}(x)\right\}$ mit folgenden Eigenschaften zu definieren:

$1^{\circ}$ Die $f_{i}(x)$ sind alle in $\langle 0,1\rangle$ erklärt und stetig.

$2^{\circ} \quad$ Es ist

$3^{\circ} \quad$ Es gilt

$$
0 \leqslant f_{i}(x) \leqslant 1 \text { für alle } i \text { und alle } x .
$$

$4^{\circ} \quad$ Für keine Folge

$$
\lim _{i \rightarrow \infty} f_{i}(x)=0 \text { für alle } x \text {. }
$$

$$
n_{1}, n_{2}, n_{3}, \ldots
$$

wachsender, natürlicher Zahlen ist gleichmäßig in $x$

$$
\lim _{k \rightarrow \infty} \frac{f_{n_{1}}(x)+f_{n_{2}}(x)+\ldots+f_{n_{k}}(x)}{k}=0 .
$$

1) Vgl. diese Studia, II (1930) pp. 51-57. Der Inhalt dieser Arbeit ist mir vor deren Niederschrift mitgeteilt worden.

$\left.{ }^{3}\right)$ Zur Behandlung dieser Frage wurde ich von Prof. Steinhaus angeregt.
Wir werden nun zunächst gewisse Hilfsmengen konstruieren, die uns dann zur Herstellung der $\left\{f_{i}(x)\right\}$ dienen werden.

Im Intervalle $\langle 0,1\rangle$ bringen wir eine, der Grösse nach wohlgeordnete, abgeschlossene Zahlenmenge $Z$ vom Typus $\omega^{\omega}+1$ unter.

Die Klammern

$(3,4),(3,5),(3,6, \ldots,(3, \infty)$,

$(4,5,6),(4,5,7) \ldots,(4,5, \infty),(4,6,7) \ldots(4,6, \infty) \ldots(4, \infty, \infty)$,

$(5,6,7,8) \ldots(5,6,7, \infty),(5,6,8, \infty) \ldots(5,6, \infty, \infty) \ldots(5,7, \infty, \infty),(5, \infty, \infty, \infty)$, $(6,7,8,9,10) \ldots \ldots$.

bilden in der hingeschriebenen Ordnung eine wohlgeordnete Menge vom Typus $\omega^{(\omega)}$.

(Man sieht, daß in (1) jede Klammer vorkommt, die mit einer natürlichen Zahl $k+1$ beginnt und $k$ natürliche Zahlen enthält:

$$
\left.k+1=n_{k+1}<n_{k+2}<\ldots \ldots \quad . . n_{2 k}\right) \text {. }
$$

Abgesehen von der grössten Zahl aus $Z$, können also die Zahlenmenge $Z$ und die Klammermenge (1) aufeinander eineindeutig (unter Bewahrung der Ordnungen) bezogen werden.

Es heiße $Z_{i}$ die Menge derjenigen Zahlen aus $Z$, welchen dabei solche Klammern entsprechen, die die Zahl $i$ enthalten.

$T_{i}$ bedeute die Menge derjenigen Zahlen aus $\langle 0,1\rangle$, die von $Z_{i}$ einen Abstand $\geqslant \frac{1}{i}$ haben.

Nun bemerkt man mit Leichtigkeit, daß die Mengen $Z_{i}, T_{i}$, $Z-Z_{i}$ alle abgeschlossen sind.

Wir setzen

$$
X_{i}=T_{i}+\left(Z-Z_{i}\right)
$$

dann ist natürlich

$$
X_{i} \times Z_{i}=0 \text {. }
$$

Da $X_{i}$ und $Z_{i}$ abgeschlossen sind, haben sie einen positiven Abstand $\delta_{i}$.

Es sei $x$ eine beliebige Zahl aus $\langle 0,1\rangle$.

Es bezeichne $\delta_{i}^{(1)}(x)$ bzw. $\delta_{i}^{(2)}(x)$ ihren Abstand von der Menge $Z_{i}$ bzw. $X_{i}$. Es ist also

$$
\delta_{i}^{(1)}(x)+\delta_{i}^{(2)}(x) \geqslant \delta .
$$


Wir setzen

$$
f_{i}(x)=\frac{\delta_{i}^{(2)}(x)}{\delta_{i}^{(1)}(x)+\delta_{i}^{(2)}(x)} \quad(i=1,2, \ldots) .
$$

Aus (2) und (3) folgt sofort die Stetigkeit von $f_{i}(x)$; wegen (3) ist auch

$$
0 \leqslant f_{i}(x) \leqslant 1 \text {, für alle } i \text { und } x .
$$

Jedes $x$ aus $\langle 0,1\rangle$ gehört nun fast allen $X_{i}$ an.

Denn gehört $x$ zu $Z$, dann gehört es zu jedem $T_{i}$, wenn nur $i>\frac{1}{\delta(x, Z)}$, wo $\delta(x, Z)$ den Abstand $x$ von $Z$ bezeichnet.

Aus (3) folgt aber

$$
f_{i}(x)=0 \text { für } x \in X_{i}
$$

und daher

$$
\lim _{i \rightarrow \infty} f_{i}(x)=0 \text { für alle } x \text {. }
$$

Es sei nun

$$
n_{1}, n_{2}, n_{3}, \ldots
$$

eine beliebige Folge wachsender, natürlicher Zahlen. $k$ sei eine beliebige, ganze Zahl.

Zufolge der bei der Definition der Klammermenge (1) gemachten Bemerkung, sieht man, daß alle $k$ Zahlen

$$
n_{k+1}, n_{k+2}, \ldots n_{2 k}
$$

immer in wenigstens einer Klammer aus (1) gleichzeitig auftreten. (Es ist ja $n_{k+1} \geqslant k+1$ ).

Es sei $x_{0}$ die dieser Klammer entsprechende Zahl aus $Z$. Es ist also

$$
x_{0} \in Z_{n_{j}} \text { für } \quad k+1 \leqslant j \leqslant 2 k
$$

und daher wegen (3)

$$
f_{n_{j}}\left(x_{0}\right)=1 \quad \text { für } \quad k+1 \leqslant j \leqslant 2 k .
$$

Dieses gibt

$$
\frac{f_{n_{1}}\left(x_{0}\right)+f_{n_{8}}\left(x_{0}\right)+\ldots+f_{n_{2 k}}\left(x_{0}\right)}{2 k} \geqslant \frac{1}{2},
$$

woraus die Behauptung $4^{\circ}$ ohne weiteres folgt.

Mit derselben Methode kann nun leicht gezeigt werden, daß wenn

mit

eine beliebige im voraus gegebene Toeplitz'sche Matrix bezeichnet, eine Funktionenfolge $\left\{f_{i}(x)\right\}$ konstruiert werden kann, die den Bedingungen $1^{\circ}, 2^{\circ}, 3^{\circ}$ genügt, und für die bei keiner Folge wachsender, natürlicher Zahlen

$$
\lim _{i \rightarrow \infty} \sum_{k=1}^{\infty} \lambda_{i k} f_{\nu_{k}}(x)=\lim _{i \rightarrow \infty} \sum_{k=1}^{i} \lambda_{i k} f_{\nu_{k}}(x)=0
$$

gleichmäßig gilt.

Mit anderen Worten, wenn man das Verfahren der ersten arithmetischen Mittel, durch ein beliebiges anderes Toeplitz'sches Summationsverfahren ersetzt, wird an der Sachlage nichts geändert. Um dies zu beweisen, erklären wir durch $r(n)$ den größten Index, für den

$$
\sum_{k=r(n)}^{n} \lambda_{n, k}>\frac{1}{2}
$$

gilt. Wegen (5) existiert natürlich ein solches $r(n)$ immer. Wegen (6) ist aber

$$
\lim _{n \rightarrow \infty} r(n)=\infty
$$

Daher gibt es bei jedem natürlichen $l$ höchstens endlich viele Indizes

für die

$$
v_{1}^{(l)}, v_{2}^{(l)}, \ldots v_{k_{l}}^{(l)}
$$

$$
r\left(\nu_{1}^{(l)}\right)=r\left(v_{2}^{(l)}\right)=\ldots=r\left(v_{k_{l}}^{(l)}\right)=l
$$

ist.

Bedeutet nun $N_{l}$ das Maximum der Zahlen

$$
\nu_{1}^{(1)}, v_{2}^{(1)}, \ldots \nu_{k_{1}}^{(1)}, \nu_{1}^{(2)}, \ldots \nu_{k_{2}}^{(2)}, \ldots \nu_{1}^{(l)}, \ldots \nu_{k_{l}}^{(l)},
$$


so können wir folgende Klammerfolge aufschreiben:

$\left(1,2, \ldots N_{1}-1, N_{1}\right),\left(1,2, \ldots N_{1}-1, N_{1}+1\right), \ldots\left(1,2, \ldots N_{1}-1, \infty\right) \ldots(1, \infty, \infty, \ldots \infty)$,

$$
\begin{aligned}
& \left(2,3, \ldots N_{2}-1, N_{2}\right) \ldots(2, \underbrace{\infty, \ldots \infty}_{N_{2}-1 \text { mal }}),
\end{aligned}
$$

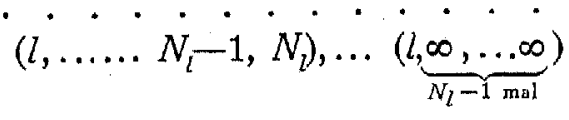

u. s. w., ganz wie in (1).

Der ganze Beweis, mit der Konstruktion der Funktionenfolge $\left\{f_{i}(x)\right\}$, kann nun wörtlich wiederholt werden, nur mul, anstatt $4^{\circ},(7)$ bewiesen werden.

Ist die Folge

$$
\nu_{1}, \nu_{2}, \nu_{3}, \ldots
$$

und die natürliche Zahl $n$ beliebig vorausgegeben, so kann man ähnlich, wie dort, in der Klammerfolge (10), eine Klammer finden, der eine Zahl $x_{0}$ aus $Z$ entspricht, für die

(11) $f_{\nu_{1}}\left(x_{0}\right)=f_{\nu_{2}}\left(x_{0}\right)=\ldots=f_{\nu_{r(n)-1}}\left(x_{0}\right)=0 ; f_{\nu_{r(n)}}\left(x_{0}\right)=\ldots=f_{\nu_{n}}\left(x_{0}\right)=1$

ist.

Aus (8) und (11) ergibt sich

$$
\begin{aligned}
\lambda_{n, 1} f_{\mathcal{\nu}_{1}}\left(x_{0}\right)+\ldots+ & \lambda_{n_{2} r(n)-1} f_{v_{r(n)-1}}\left(x_{0}\right)+\lambda_{n, r(n)} f_{\nu_{r(n)}}\left(x_{0}\right)+\ldots \\
& +\ldots+\lambda_{n, n} f_{\nu_{n}}\left(x_{0}\right)>\frac{1}{2}
\end{aligned}
$$

was (7) beweist.

(Reçu par la Rédaction le 20. 12. 1929).

\section{Sur une propriété du champ des fonctions continues}

par

Z. ZALCWASSER (Varsovie).

1. Le but de cette note est la résolution d'un problème posé par M. S aks concernant les suites convergentes de fonctions continues.

$$
\text { Soit } \quad f_{1}(x), f_{2}(x), \ldots f_{n}(x), \ldots
$$

une suite des fonctions vérifiant les conditions suivantes:

a) $\lim _{n \rightarrow \infty} f_{n}(x)=f(x)$ pour tout $x \in(a, b)=I$,

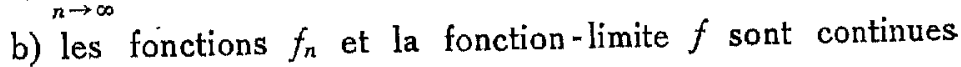
dans $I$,

c) $\left|f_{n}(x)\right| \leqslant M$ pour tout $n$ naturel et $x \in I$.

Appelons moyenne générale de la suite (1) toute expression de la forme:

$$
\varphi(x)=\lambda_{1} f_{n_{1}}(x)+\lambda_{2} f_{n_{2}}(x)+\ldots+\lambda_{k} f_{n_{k}}(x)
$$

où $\lambda_{i}$ sont des constantes telles que

$$
\lambda_{i} \geqslant 0(i=1,2, \ldots k), \quad \sum_{i=1}^{k} \lambda_{i}=1 .
$$

La question posée par M. Saks est la suivante:

„La suite (1) vérifiant les conditions a), b), c), peut on à chaque $\varepsilon>0$ faire correspondre une moyenne générale $\varphi(x)$ de la suite (1), de manière que

$$
|\varphi(x)-f(x)| \leqslant \varepsilon \text { pour tout } x \in I ? " “
$$

La réponse est affirmative et nous en allons donner une démonstration.

2. Nous nous servirons de la notion de l'indice de la suite (1) dans un intervalle. Soient 